\title{
On the study of predictors in Single Shot Multibox Detector
}

\author{
Xuemei Xie, Xun Xu, Lihua Ma, Guangming Shi, Pengfei Chen \\ School of Artificial Intelligence \\ Xidian University \\ Xi'an, China \\ xmxie@mail.xidian.edu.cn
}

\begin{abstract}
Single shot multibox detector (SSD) is a state-of-the-art network for real-time object detection. It is originally designed for general datasets. While, for specific datasets, their distribution of ground truth boxes is somehow different and thus, SSD shows unsatisfying performance. In this paper, we improve the performance of SSD on specific datasets. We first dissect the mechanism of predictors, the predicting parameters of a potential detection, in two aspects: classification and localization. Then we reveal the relationship between default boxes and predictors. With this point we finally make an improvement on default box setting and achieve a higher mAP over the original SSD on specific datasets.
\end{abstract}

\section{CCS Concepts}

- Computing methodologies $\rightarrow$ Artificial intelligence $\rightarrow$ Computer vision $\rightarrow$ Computer vision problems $\rightarrow$ Object detection.

\section{Keywords}

predictor; SSD; default box; general datasets; specific datasets.

\section{INTRODUCTION}

Object detection is a challenging task including classification [1] [2] and localization. SSD [3] is a network for general object detection. Unlike other detection networks that based on the generation of region proposals [4] [5] [6] [7], SSD network eliminates the region proposals and outputs the detection results directly, like YOLO [8]. What's more, it sets default boxes on scale-variant feature maps to cover objects in various sizes. As a result, SSD outperforms most existing object detection networks.

The standard SSD is designed for general datasets such as PASCAL VOC. For specific datasets, SSD still needs to be further improved, owing to the difference with VOC datasets. Default box is an essential part in SSD. Each kind of default boxes is corresponding to a classification predictor and a localization predictor that output the detection results. In this paper, we give an implicit declaration of the important role that those predictors play. And we also pay attention to the relationship between the default box setting and the quality of those predictors. Different

Permission to make digital or hard copies of all or part of this work for personal or classroom use is granted without fee provided that copies are not made or distributed for profit or commercial advantage and that copies bear this notice and the full citation on the first page. Copyrights for components of this work owned by others than ACM must be honored. Abstracting with credit is permitted. To copy otherwise, or republish, to post on servers or to redistribute to lists, requires prior specific permission and/or a fee. Request permissions from Permissions@acm.org. ICVIP'17, December 27-29, 2017, Singapore, Singapore. Copyright 2017 ACM ISBN 978-1-4503-5383-0/17/12 ..\$15.00 DOI: https://doi.org/10.1145/3177404.3177412 from region proposals in some detection networks, e.g. Faster R$\mathrm{CNN}$, which provide an "attention" mechanism to guide the network where to look, default boxes in SSD are utilized to discretize the possible output space. In fact, these default boxes decide which training samples contribute to both classification and localization predictors. The classification predictor is actually a logistic regress or which outputs the confidence score of each object class, while the localization predictor is a linear regress or which outputs the coordinate information of each object. The quality of those two predictors not only depends on the base classification network [9], but also highly depends on the quantity of the training samples which are used to train those predictors.

In order to get a further improvement on a specific object detection, we need to redesign the distribution of default boxes in SSD network. Our experiments show that predictors, no matter classification predictor or localization predictor, give a pretty good performance when there are enough training samples scattering around the corresponding default box. As a result, the proposed SSD shows great detection results on specific datasets.

\section{OVERALL FRAMEWORK}

SSD has proposed a method for object detection using multi-scale features of images. The detection task is implemented by two parts: feature extraction and prediction, which is shown in Figure 1. The prediction module consists of two components: the classification predictor and the localization predictor, as the yellow block and the blue block show. The feature extraction module uses six convolution layers to extract deep features of images for both classification and localization task. Then, the classification predictor can give the result of confidence score of latent objects, and the localization predictor predicts the location offsets of default box.

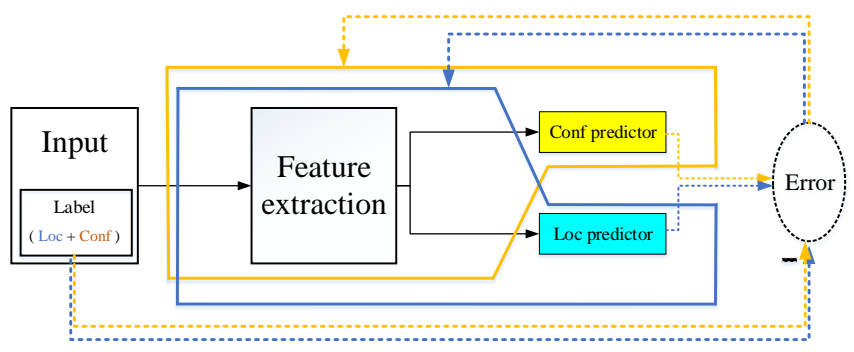

Figure 1. The overall training framework of SSD includes feature extraction and predictors for two tasks (localization and classification). The two predictors share the same feature extracted from conv layers.

In Figure 1, dotted lines indicate the training phase, in which parameters are supervised by given label information of input images. The network is updated by back propagating the errors 
between label information and prediction results. For the classification task, the error between the confidence score and class label propagates to tune the confidence predictor and the feature extraction module, which is shown in the frame in yellow solid line. For the localization task, the error between the location offsets and ground truth boxes is used to adjust the localization predictor and the feature extraction as the frame in blue solid line shows. In order to predict bounding box of accurate location and high confidence score, the loss function minimizes the weighted sum of the location loss and the confidence loss,

$$
L=\frac{1}{N}\left(L_{c o n f}(x, c)+\alpha L_{l o c}(x, l, g)\right)
$$

where $\mathrm{N}$ is the number of matched default boxes, $\mathrm{x}$ is the match index, $\mathrm{c}$ is the confidence score, 1 is the prediction and $\mathrm{g}$ is the ground truth. We will introduce matching strategy in later sections. Both two losses contribute to their corresponding predictors respectively. At the beginning of training phase, predictors are initialized with random Gaussian values. After times of iteration, predictors can be well trained to be adaptive to training dataset and they can show great performance in testing phase.

As we know, the classification predictor solves a logistic regression problem for its discretized category information while the location predictor is trained to establish a linear regression for its successive output of bounding boxes. The logistic regression requires predictor to distinguish features, while linear regression requires predictor to establish linear relationship between independent variable and dependent variable. During training time, these selected samples will act on both two tasks to supervise the training of predictors.

\section{TRAINABLE PREDICTORS}

The original SSD is a general network with multi-scale feature maps. The feature extraction of SSD consists of six convolution layers: conv4_3, fc7, conv6_2, conv7_2, conv8_2, pool6. For simplicity, we analyze and discuss the mechanism and effectiveness of predictors according to a single scale feature layer, conv4_3 layer. The output of feature extraction is considered as the input of prediction task. In predicting phase, predictors utilize features to predict the box offset relative to specific default box and confidence score of each object. Predictors in two tasks share the same feature extraction module. We will introduce the major role predictors play in both two tasks: classification and regression.

\subsection{Predictor for classification}

The classification task, mentioned as the frame in yellow solid line in Figure 1 is described specifically in Figure 2. It aims to separate multiple classes according to discriminative feature from conv4_3. In this task, the predictor for classification is a set of filters with the filter size being $3 \times 3 \times 512$, which works like the last fully connected layer in classification network. However, compared with traditional classification network, it extracts local features for object scoring. The number of filters depends on the number of categories and the number of default boxes in each feature map cell. Default boxes from each feature map cell are shared throughout the whole feature map. With this mechanism, SSD can predict objects in different locations in an image. The confidence predictor learns to distinguish features of multiple classes according to a confidence loss function.

The matching strategy of SSD in Figure 2 is crucial to the performance of predictors, which is something similar to training sample selection. For both two tasks, the network with supervised training needs plenty of robust training samples. Matching strategy is applied when default boxes of different aspect ratios are generated from each feature map cell. Default boxes which have a large overlap with a ground truth will inherit the information of the ground truth box including its location and category of object. It is a procedure of selecting sample for default box.

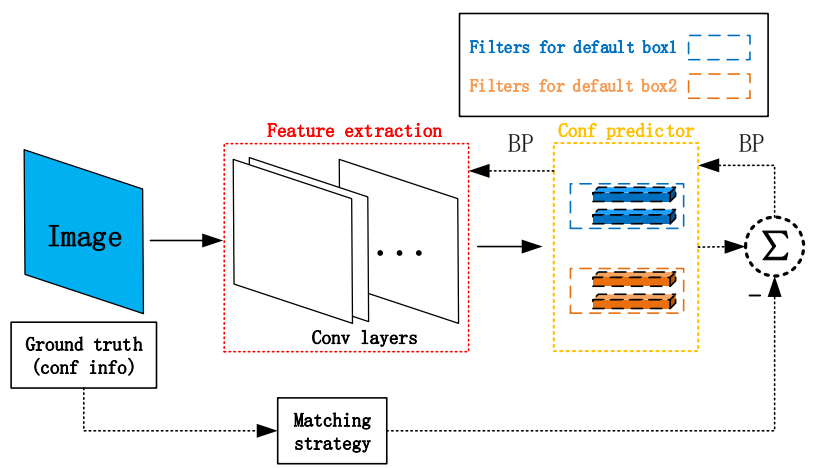

Figure 2. The structure of two classes training of confidence predictor, where only two classes and two default boxes are shown for simplicity. Each default box corresponds two filters. Each filter of a default box is responsible for the confidence prediction of a single class. This structure details how the confidence error are generated and back propagated to tune the confidence predictor and the whole network.

The confidence loss is softmax loss over multiple classes score:

$$
L_{\text {conf }}(x, c)=-\sum_{i \in \text { Pos }}^{N} x_{i j}^{p} \log \left(\hat{c}_{i}^{p}\right)-\sum_{i \in N e g} \log \left(\hat{c}_{i}^{0}\right)
$$

where $x_{i j}^{p}$ is 1 if $i$-th default box matches $j$-th ground truth, reversely, it is $0 . c_{i}^{p}$ is the output of confidence predictor as shown in Figure 3. During training time, the matching strategy divides default boxes into positive and negative samples. All positive samples will be labeled as the label of ground truth and negative ones will be labeled as background. Then the predictor can be trained under the supervision of these label information. After times of iterations, filter $\theta_{k}^{p}$ corresponding to $k$-th default box will be trained to score a high confidence on the feature of $p$ th class.

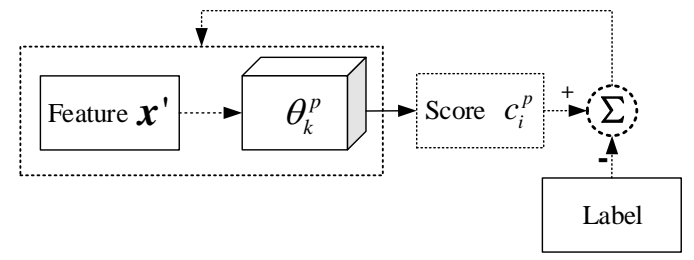

Figure 3. Training of filters in confidence predictor.

In VOC dataset, the aspect ratios of ground truth samples are generally distributed as shown in Figure 4. As we can see in this figure, the setting of default box in original SSD has fitted all these samples. For each default box, those surrounding samples will contribute to train its corresponding predictors. 
It is worthwhile to note that the actual distribution is a little bit different because of the data augmentation in SSD. In reality, augmentations such as shift, translation and so on make the model robust to various object sizes and shapes.

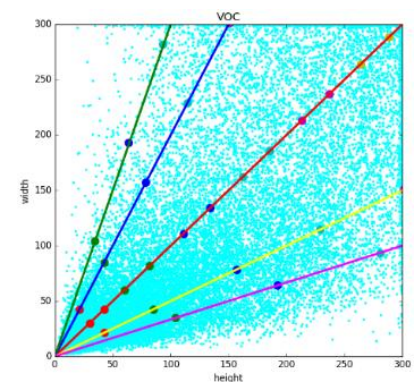

Figure 4. Distribution of samples with various aspect ratios in VOC dataset. One line represents an aspect ratio. The colored points on lines indicate the aspect ratio of default box with different scales. Points with same color come from the same feature layer. All those scattered points are objects in VOC.

Default boxes uniformly discretize the space of ground truth, but this case is different in single class. The distribution of two class (sofa and bird) is in Figure 5. We focus on the distribution in conv4_3 layer. It shows that there are fewer sofa samples than bird samples in conv4_3. Since samples are essential to train a classifier or a predictor, the predictor for bird can be trained better than sofa. The filter for bird is better fitting to bird samples and it can easily score a high confidence when it comes a bird's feature.

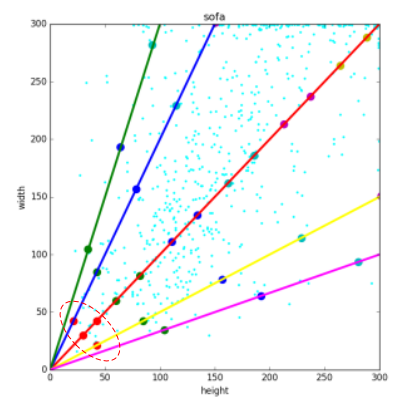

(a) Distribution of sofa

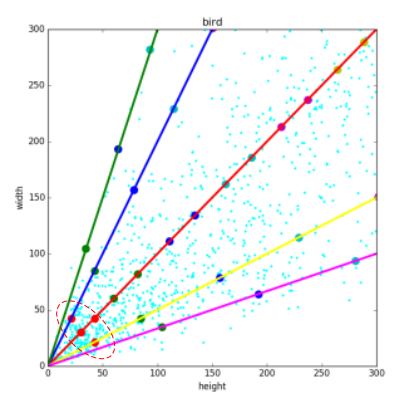

(c) Distribution of bird

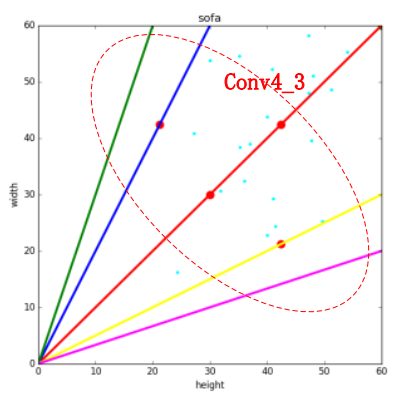

(b) enlarged region in conv4_3 of (a)

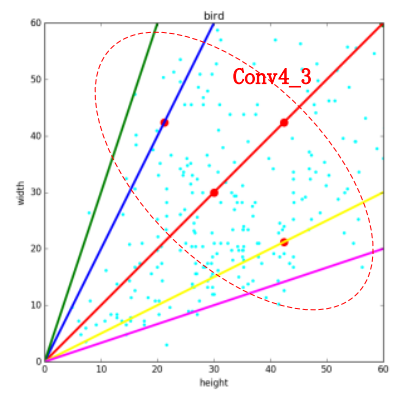

(d) enlarged region in conv4_3 of (c)
Figure 5. Two classes object distribution of aspect ratios of samples in conv4_3. The figure in right shows the enlarged region of conv4_3.
The experiment results are shown in Figure 6. Score of sofa is lower than that of bird because default boxes in conv4_3 are surrounded by more bird samples and for bird performs better than for sofa.

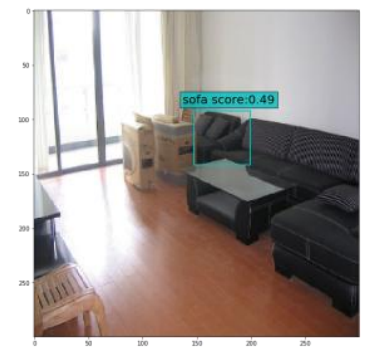

(a) Sofa

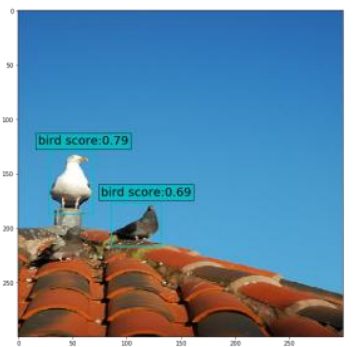

(b) Bird
Figure 6. The confidence score of objects in two classes.

\subsection{Predictor for regression}

In localization task, predictor aims to find a linear relationship between feature and location information. It is mentioned as the frame in blue solid line in Figure 1 and described specifically in Figure 7. It predicts the box offset of each default box. After matching strategy, the space of ground truth boxes is divided by default boxes, so each default box has its own positive samples. These samples supervise the training of the predictor. At the end of training phase, the linear regression model is established and default box regresses to its adjacent ground truth with the offset it has learned.

Localization loss uses a Smooth L1 loss for bounding box regression. It minimizes the error between the predicted box offset $\left(l_{i}^{m}\right)$ and ground truth box offset $\left(\hat{g}_{j}^{m}\right)$ :

$$
L_{l o c}(x, l, g)=\sum_{i \in P o s}^{N} \sum_{m \in\left\{c_{x}, c_{y}, w, h\right\}} x_{i j}^{k} \operatorname{smooth}_{L 1}\left(l_{i}^{m}-\hat{g}_{j}^{m}\right)
$$

As for conv4_3 layer, prediction $l_{i}^{m}$ is the convolution of conv4_3 feature $x^{\prime}$ and filter $\theta_{k}^{m}$ of $k$-th default box. $\theta_{k}^{m}$ aims to mapping feature to the box offset relative to default box $k$ as shown in Figure 8.

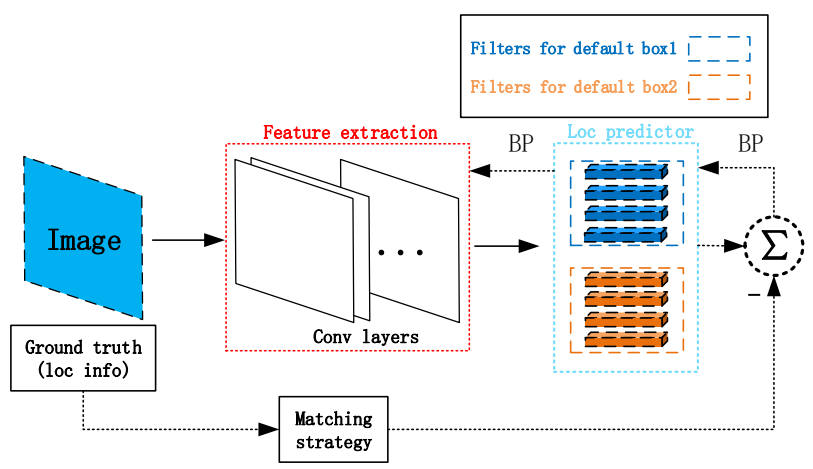

Figure 7. The structure of the training of localization predictor. Localization predictor includes 4 filters for each default box in order to predict location information $(x, y$, w, h) of bounding box. 


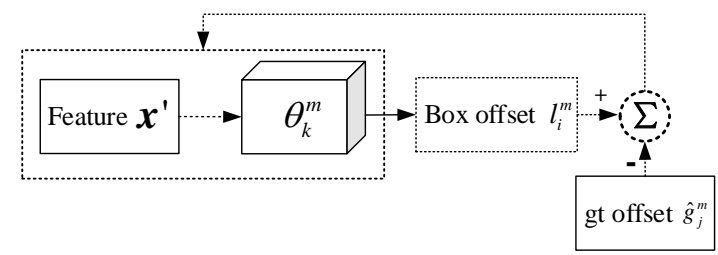

Figure 8. Training of filter in localization predictor.

In regression task, training data is quite important for training a predictor with good performance. Only positive samples chosen from matching strategy have been used for training. Each default box will match a specific set of ground truth boxes, which indicates that a specific linear regression relationship will be established between the feature and box offset as shown in Figure 9. Figure 9(a) shows an underfitting case where lack of default box is hard for predictor to establish such relationship. If it is too much as shown in Figure 9(c), it is an overfitting case where such relationship only performs well on training data. As a result, default box setting depends on the distribution of samples. If we set default box reasonably like Figure 9(a), linear regression relationship will be established appropriately.
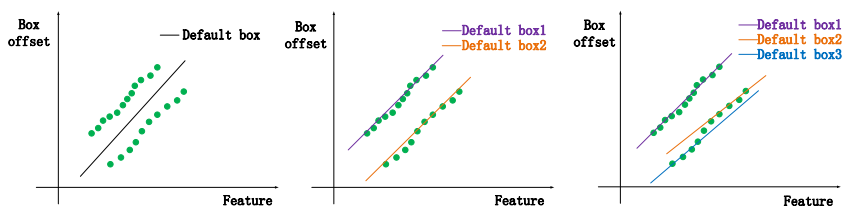

Figure 9. The three cases distribution of ground truth box in the relationship between feature extracted from conv4_3 layers and box offset.

\section{EXPERIMENTS}

In this section, we first test single class object in VOC dataset with different sizes. Then we compare the distribution of two classes object in VOC and test the performance on both of them. Finally we make an improvement on default box setting on our specific motor dataset [11]. For our dataset, we conduct experiment using both original default box setting and our redesigned default box setting. The system run on the PC is equipped with Core i7-4790 CPU, 3.60GHz frequency and 8GB RAM. And all of these experiments are all conducted on the platform of Caffe [10].

\subsection{Experiments on VOC single class}

This section shows experiments on VOC single class dataset including two parts: small and large object, multiple shapes.

Small and large object For a single class, the small objects in Figure 10 are predicted low scores because their surrounding default boxes are too 'far' from them in Figure 5. However, the larger ones are score high confidence as they are surrounded by default boxes. These small objects are considered outliers and predictor can hardly respond to them. Naturally we attempt to set default box on a relatively small scale such as conv3_3. However, such change introduce a higher computational complexity because feature map in conv3_3 is larger.

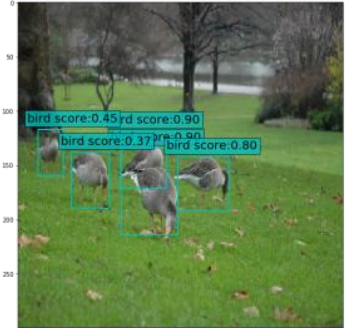

(a) Large objects

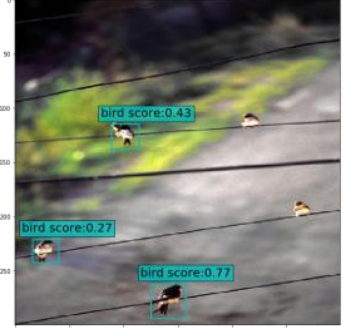

(b) Small objects
Figure 10. The confidence scores of both small and large objects.

Multiple shapes According to the distribution of two classes object in conv4_3 shown in Figure 11, the ground truth of aeroplane is mostly distributed in horizontal direction while bottle is distributed in vertical direction. Such distinction causes training intensity difference over predictors of different default boxes, and naturally causes prediction difference.

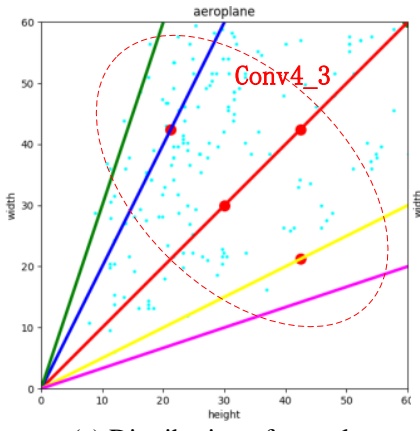

(a) Distribution of aeroplane

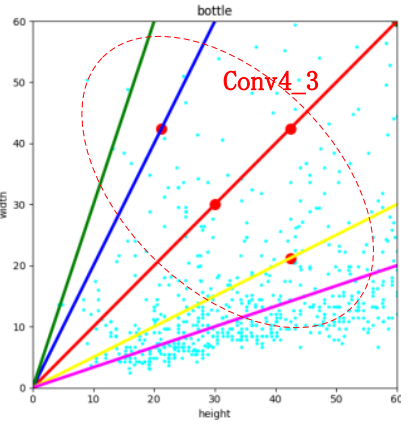

(b) Distribution of bottle
Figure 11. The distribution of two classes object in conv4_3.

We show the detection results on both two classes. For a single class, the results come from default boxes with different aspect ratios. As shown in Figure 12 and Figure 13, the predicted boxes (magenta box) are regressed from default boxes (blue box). For aeroplane, the horizontal default box performs better than vertical default box in both score and location because samples of aeroplane are mostly horizontal direction. While for bottle, the vertical default box is better because its samples are mostly distributed in vertical direction.

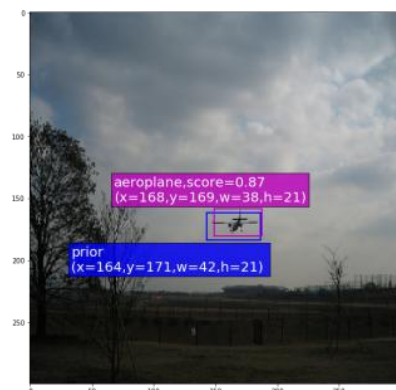

(a) Horizontal default box

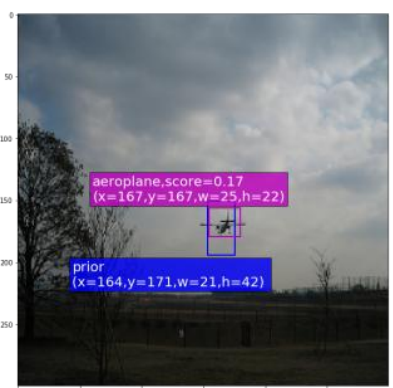

(b) Vertical default box
Figure 12. Detection results of aeroplane. 


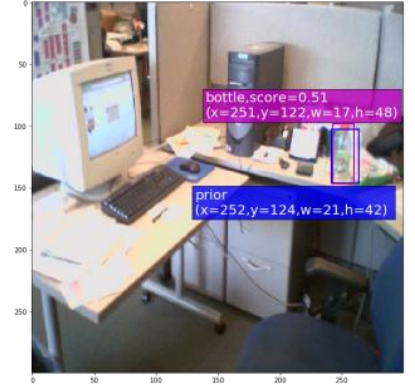

(a) Horizontal default box

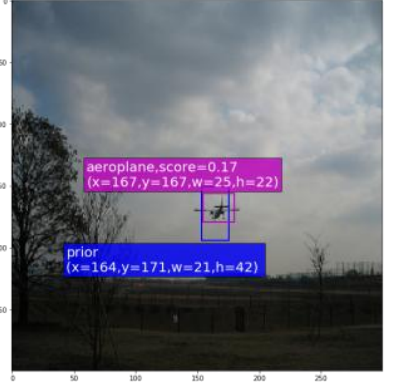

(b) Vertical default box
Figure 13. Detection results of bottle.

\subsection{Specific datasets}

In this section, we only design our SSD model for detecting motorbike objects in city traffic road. We construct our specific vehicle dataset [11] by taking traffic videos directly in Xi'an city, Shanxi province. In these video captions, we only focus on the motorbike single class with varies colors, shapes, and luminance.

According to specific dataset, we find that original default box setting is not fit to train the predictors. So detection results show poor performance on both confidence and localization. The original default box setting is not for the distribution of motor dataset. This distribution needs a set of default boxes that could finely cover those samples. With this motivation, we set default box as Figure 14 where default boxes can cover the whole motor dataset. In detail, we change the original default box aspect ratios 2 to 3 in conv4_3 like the red points. All the blue points are the original default boxes in standard SSD.

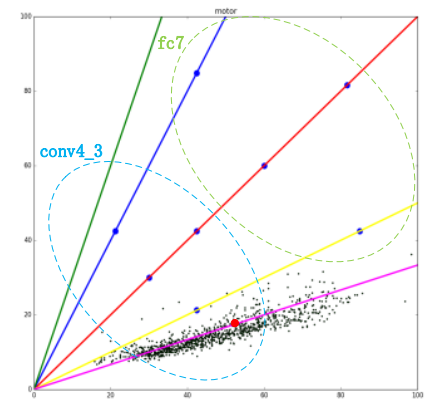

Figure 14. The distribution of motor dataset in conv4_3 and our default box setting.

We test both the original and the improved model on motor dataset. As shown in Figure 15, the detection results including confidence scores of objects and width and height of predicted boxes on two models. The original model scores low confidence on the motor objects while our improved model scores a higher confidence. This is because predictors in improved model is learned better. There are more samples surrounding those default boxes we design. And it introduces less outliers to predictor. With this change, our model outperforms the original one with mAP $2.81 \%$. So the proposed model can get a better detection result.

\section{CONCLUSION}

This paper gives a view of understanding the detection mechanism of SSD network both in classification and localization. It shows the important role default boxes plays in training the classification and localization predictors. SSD utilizes different default boxes for choosing training samples for those predictors. More or less default boxes setting, even unsuitable default boxes setting in shapes, would influence the quantity of samples contributing to the training of predictors. Our experiments show the importance of default boxes in detecting objects. We finally draw a conclusion that default box setting should follow the distribution of aspect ratios of training samples.

In multi-classes task, sometimes there exists sample imbalance. The categories with few training sample show pool performance in both extracting features and training predictors. In the future work, we are going to fuse handcrafted features and $\mathrm{CNN}$ features of categories that lack of training samples. With the fused features, we could design specific predictors artificially, which would help to deal with few sample case.

\section{ACKNOWLEDGMENTS}

This work is supported by Natural Science Foundation (NSF) of China (61472301) and Ministry of Education project (6141A02011601).

\section{REFERENCES}

[1] Krizhevsky A, Sutskever I, Hinton G E. "ImageNet classification with deep convolutional neural networks," International Conference on Neural Information Processing Systems. Curran Associates Inc. 1097-1105, (2012).

[2] Simonyan K, Zisserman A. "Very Deep Convolutional Networks for Large-Scale Image Recognition," Computer Science, (2014).

[3] Liu, W., Anguelov, D., Erhan, D., Szegedy, C., Reed, S., Fu, C. Y., \& Berg, A. C. "Ssd: Single shot multibox detector," In European conference on computer vision. 21-37, (2016).

[4] R. Girshick, J. Donahue, T. Darrell, and J. Malik, "Rich Feature Hierarchies for Accurate Object Detection and Semantic Segmentation," in IEEE Conference on Computer Vision and Pattern Recognition. 580-587, (2014).

[5] He, K., Zhang, X., Ren, S., \& Sun, J. Spatial pyramid pooling in deep convolutional networks for visual recognition. In European Conference on Computer Vision. 346-361, (2014).

[6] R. Girshick, "Fast R-CNN," IEEE International Conference on Computer Vision. 1440-1448, (2015).

[7] S. Ren, K. He, R. Girshick, and J. Sun, "Faster R-CNN: Towards Real-Time Object Detection with Region Proposal Networks," IEEE Transactions on Pattern Analysis and Machine Intelligence. 91-99, (2016).

[8] Redmon, J., Divvala, S., Girshick, R., \& Farhadi, A. You only look once: Unified, real-time object detection. In Proceedings of the IEEE Conference on Computer Vision and Pattern Recognition. 779-788, (2016).

[9] He, K., Zhang, X., Ren, S., \& Sun, J. Deep residual learning for image recognition. In Proceedings of the IEEE conference on computer vision and pattern recognition, 770778, (2016).

[10] Jia, Yangqing, Shelhamer, Evan, Donahue, Jeff, Karayev, Sergey, Long, and Jonathan, "Caffe: Convolutional Architecture for Fast Feature Embedding," Acm International Conference on Multimedia. 675-678, (2014).

[11] http://see.xidian.edu.cn/faculty/gmshi/dataset.htm 

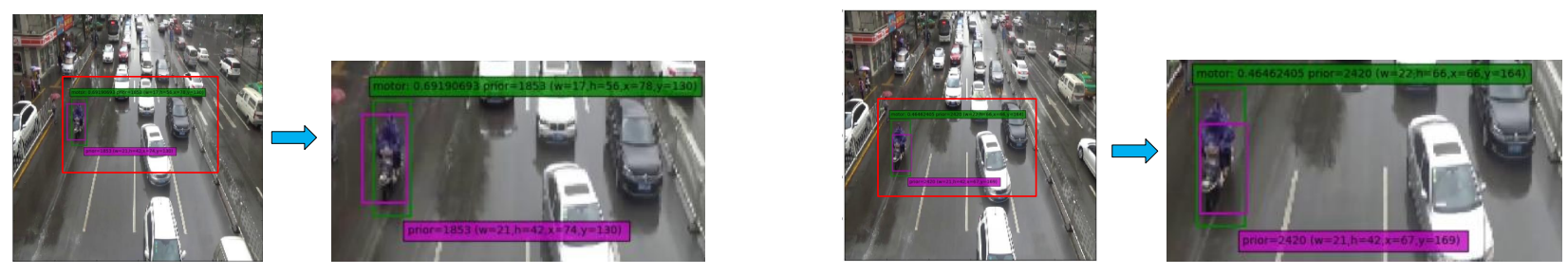

(a) Original model
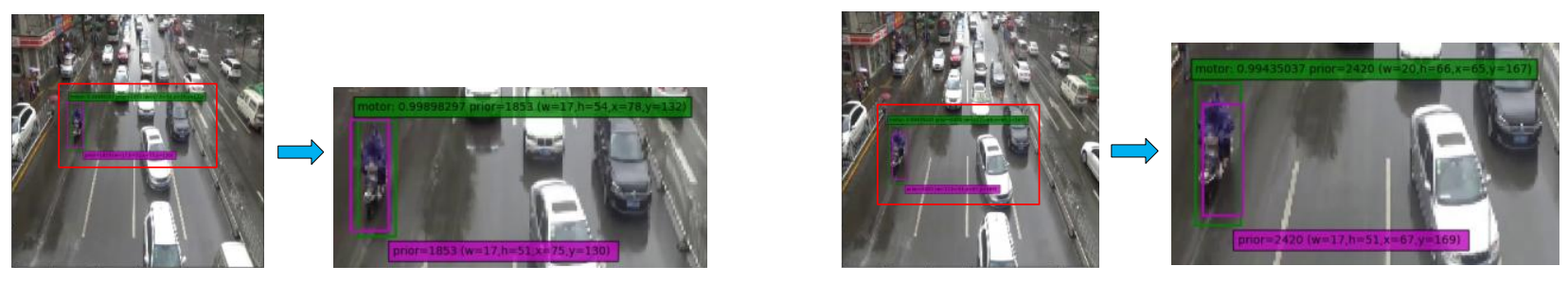

(b) Proposed model

Figure 15. Detection results on the original model and our improved model on motor dataset. 